АНАЛИЗ ПРОСТРАНСТВЕННОЙ СТРУКТУРЫ И СПЕКТРА ЖЕСТКОГО РЕНТГЕНОВСКОГО ИЗЛУЧЕНИЯ ВСПЫШКИ 23 СЕНТЯБРЯ 2014 Г. ПО ДАННЫМ НАБЛЮДЕНИЙ RHЕSSI, SDO, NOBEYAMA RADIOHELIOGRAPH

\author{
Овчинникова Е.П. ${ }^{1}$, Шабалин А.Н. ${ }^{1}$, Глобина В.И. ${ }^{2}$, Чариков Ю.Е. \\ ${ }^{1}$ ФТИ им. А.Ф. Иоффе РАН, г. Санкт-Петербург, Россия \\ ${ }^{2}$ Главная (Пулковская) астрономическая обсерватория РАН, Санкт-Петербург, Россия
}

\title{
ANALYSIS OF SPATIAL STRUCTURE, HARD X-RAY SPECTRUM IN THE FLARE OF SEPTEMBER 23, 2014, BASED ON RHESSI, SDO, NOBEYAMA RADIOHELIOGRAPH OBSERVATIONS
}

\author{
Ovchinnikova E.P. ${ }^{1}$, Shabalin A.N. ${ }^{1}$, Globina V.I. ${ }^{2}$, Charikov Yu.E. ${ }^{1}$, \\ ${ }^{1}$ Ioffe Institute, RAS, St. Petersburg, Russia \\ ${ }^{2}$ Central Astronomical Observatory at Pulkovo of RAS, St. Petersburg, Russia
}

An analysis of 09/23/2014 23:11UT solar flare, GOES-class M2.5 is presented. The radio (Nobeyama Radioheliograph) and X-ray (RHESSI) radiation maps, their temporal and spatial evolution were studied in detail. The magnetic field in the chromosphere and the corona of the active region were extrapolated. Based on radio and X-ray dynamics, it is convenient to divide magnetic arcade into two parts - the northern and the southern. The northern part is a magnetic loop with the ratio $B_{\max } / B_{0} \sim 7$ in the north footpoint and $\sim 1.8$ in the south footpoint $\left(B_{0} \sim 120 G\right)$. The southern part represents a set of loops extending from north to south and connecting large magnetic spots with the ratios $B_{\max } B_{0} \sim 25$ in the north and $B_{\max } / B_{0} \sim 9$ in the south. The time profile has a complex structure: the build up phase, a sequence of three peaks growing in amplitude and a decay phase. During the build up phase, two sources of hard X-rays (25-50 keV) are observed in the northern and southern parts of the arcade. X-ray sources are associated with radiation from the southern footpoints of the loops. The absence of sources in the northern part of the loops is probably caused by a strong asymmetry of the loop magnetic field. The position of radio emission sources $(17,34 \mathrm{GHz})$ coincides with the regions of the minimum of the magnetic field. At the moment of the main peak, the source of hard X-rays in the northern part of the arcade was associated with the footpoint of the north magnetic loop.

\section{DOI: 10.31725/0552-5829-2018-315-318}

Обработка и интерпретация жесткого рентгеновского и гиросинхротронного излучения солнечных вспышек позволяют модельно восстановить параметры ускоренных электронов (поток, спектр, поляризацию) и плазмы - концентрацию, возможно размер области излучения. Следует заметить, что указанные параметры характеризуют пучок и плазму только в момент излучения, но не в момент инжекции и, тем более, в момент ускорения. При этом, удается восстановить вид распределения электронов только по энергии, распределение по питч-углам остается неизвестным [1, 3]. В моделях, учитывающих кинетику процессов распространения элек- 
тронов, ввиду сложности расчетов, рассматриваются упрощенные магнитные структуры вспышечных петель [2]. В данной работе проведен анализ наблюдательных данных комплексной структуры во вспышечной области, которую нельзя свести к однопетлевому приближению, с целью дальнейшего моделирования кинетических процессов для рассматриваемого события.

\section{Конфигурация магнитного поля и динамика локальных источников излучения вспышки SOL2014-09-23}

По магнитограммам HMI/SDO [6] в пакете GX_SIMULATOR [5] была восстановлена геометрия магнитного поля в хромосфере и короне Солнца на момент развития вспышки SOL2014-09-23. Восстановленная структура представляет собой совокупность магнитных петель, образующих аркаду (рис. 1). Исходя из различия в эволюции локальных источников регистрируемого радио и рентгеновского излучений, представим аркаду как состоящую из северной и южной частей. Северная часть представляет собой магнитную петлю с отношением $\mathrm{B}_{\max } / \mathrm{B}_{0} \sim 7$ на севере и 1.8 на юге $\left(\mathrm{B}_{0}=121\right.$ Гс). Южная часть - совокупность петель, протянувшихся с севера на юг и соединяющих крупные, разнополярные пятна с чрезвычайно большим градиентом магнитного поля - отношения $\mathrm{B}_{\max } / \mathrm{B}_{0} \sim 25$ на севере и $\mathrm{B}_{\max } / \mathrm{B}_{0} \sim 9$ на юге. Одной из важных особенностей данного события является динамика жесткого рентгеновского излучения (ЖРИ): локальные источники (данные RHESSI) наблюдаются в разные моменты времени, максимумы интенсивности которых приходятся на разные фазы вспышки.

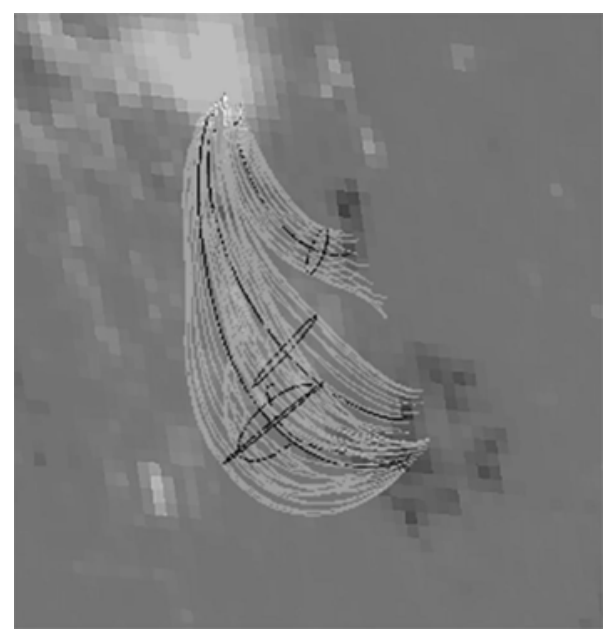

Рис. 1. Фоновое изображение - магнитограмма HMI/SDO и магнитные петли, полученные методом LFFF. Север - в верхней части рисунка.

На фазе роста потока наблюдаются два источника ЖРИ (рис. 2) в северной и южной частях аркады. Источники ассоциированы с излучением из южных оснований петель. Отсутствие зеркальных северных источников в основаниях вызвано сильной асимметрией магнитного поля и анизотро- 
пией ускоренных электронов в момент инжекции. Положение источника радиоизлучения $(17,34$ ГГц) [3] совпадает с областями минимума магнитного поля для северной магнитной петли. Максимум потока для северного источника ЖРИ (рис. 2) наблюдается в момент второго пика рентгеновского излучения, а энергетический спектр, рассчитанный с учетом альбедо, аппроксимируется степенным законом с показателем $\gamma \sim 2.7$.
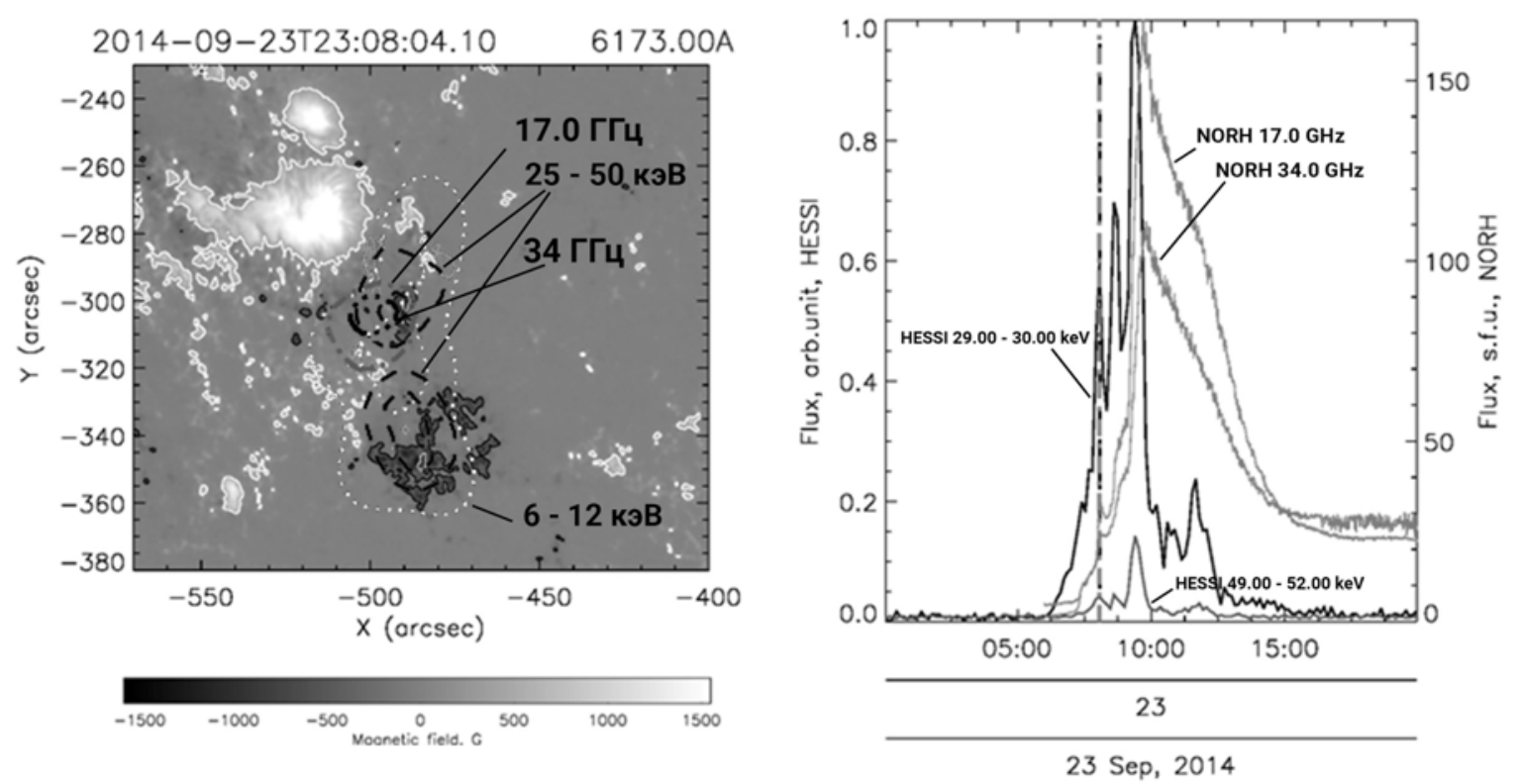

Рис. 2. Для момента времени первого пика ЖРИ: слева - магнитограмма HMI/SDO (контуры на уровне 275 Гс). Поверх магнитограммы нанесены контурные линии жесткого рентгеновского (6-12, 25-50 кэB, RHESSI) и радио излучения (17, 34 ГГц, NORH). Справа: скорости счета 29-30 кэВ, 49-52 кэВ и поток на частотах 17 ГГц, 34 ГГц.
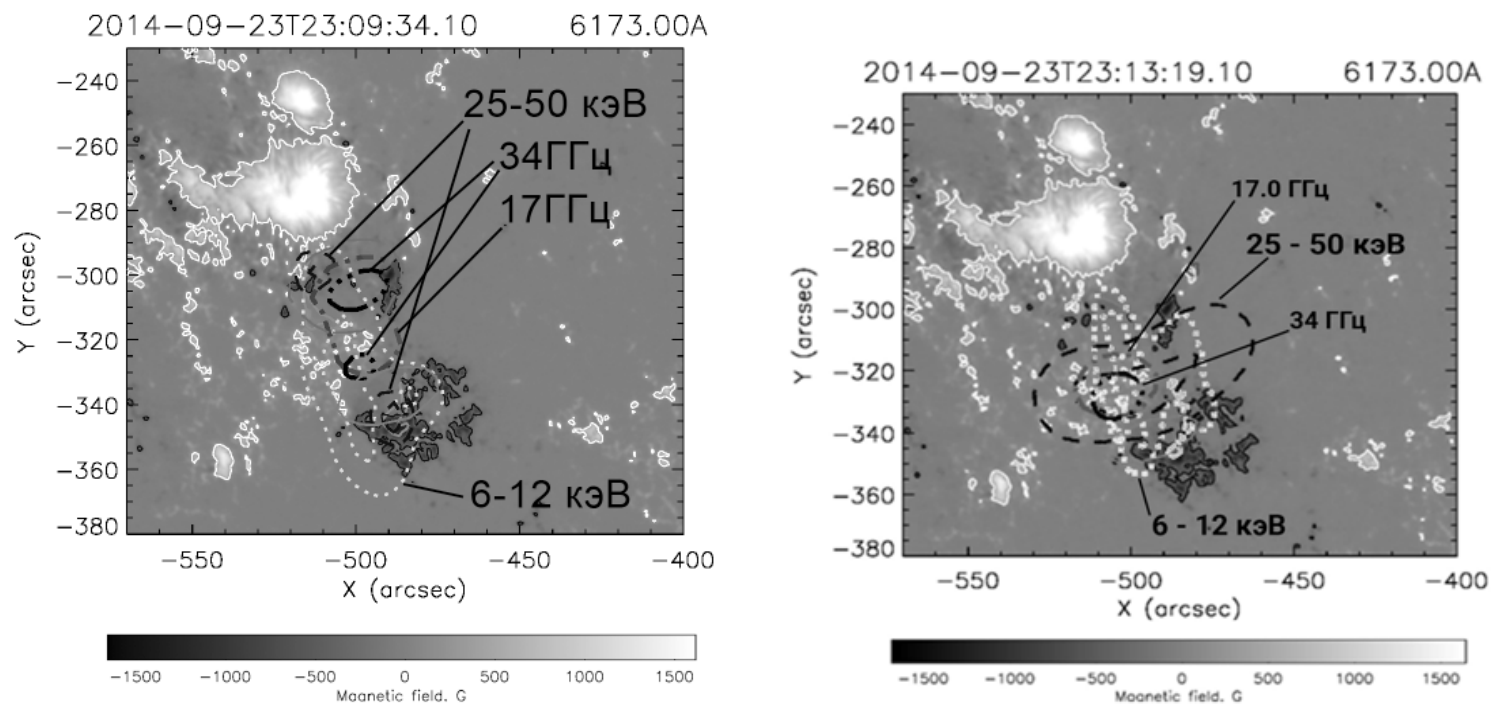

Рис. 3. Магнитограмма и контуры ЖРИ (описание см. рисунок 2).

Слева: в момент главного пика, справа: на фазе спада рентгеновского излучения.

На фазе главного пика (рис. 3. слева) для источника ЖРИ южной части аркады наблюдается максимум излучения, показатель спектра с учетом 
альбедо $\gamma \sim 3.0$. В северной части аркады источник ЖРИ смещается, его положение невозможно однозначно ассоциировать с конкретной областью магнитной петли. Данный источник может быть локализован как в вершине северной петли, так и в основании одной из петель южной части аркады. Подробнее этот вопрос рассматривается при моделировании радио и рентгеновского излучения данного источника [7]. Радио излучение локализовано в двух областях, положение которых соотносится с минимумами магнитного поля северной и южной частей аркады.

На фазе спада (рис. 3, справа) локализация рентгеновских и радио источников соответствует картине захвата электронов в ловушку в области минимума магнитного поля - то есть в верхней части петли.

\section{Заключение}

Проделанный анализ локализации и динамики источников ЖРИ и радиоизлучения в восстановленной конфигурации магнитного поля для вспышки SOL2014-09-23 позволяет перейти к следующему этапу работы моделированию процессов распространения ускоренных электронов с целью определения параметров пучка и плазмы, как в момент инжекции, так и в последующие моменты, связанные с излучением ЖРИ и гигагерцового радиоизлучения [7].

Работа Овчинниковой Е.П., Шабалина А.Н. и Глобиной В.И. поддержана грантом РФФИ мол_а № 18-32-00405

\section{Литература}

1. Brown J.C. // Solar Physics. 1971. № 3 (18). C. 489-502.

2. Charikov Y.E., Shabalin A.N., Kuznetsov S.A. // Geomagnetism and Aeronomy. 2017. № 8 (57).

3. Huang G., Nakajima H. // The Astrophysical Journal. 2009. № 1 (696). C. 136-142.

4. Ning Z., Cao W. // Solar Physics. 2009. № 2 (257). C. 335-350.

5. Nita G.M. et al. // The Astrophysical Journal. 2015. № 2 (799). C. 236.

6. Schou J. et al. // Solar Physics. 2012. № 1 (275). C. 229-259.

7. Шабалин А.Н. и др. // XXII Всероссийская ежегодная конференция по физике Солнца «Солнечная и солнечно-земная физика - 2018». Труды. ГАО, СПб, 2018. 